\title{
Reflexiones acerca de las potencialidades y limitaciones de la inclusión social
}

\author{
Lisandro Fernández* \\ Universidad de Buenos Aires-CONICET, La Plata, Argentina
}

\section{RESUMEN}

El presente trabajo tiene como finalidad principal contribuir al debate sobre la noción de inclusión social, la cual se insertó en las últimas décadas como objetivo central de las políticas públicas, asociada con una connotación positiva. Si bien la inclusión social se caracteriza por su elevado grado de aceptación política y social, en el campo académico se señala su excesivo nivel de generalidad y escasa densidad teórica. En ese marco, el artículo realiza una reflexión teórica acerca de las potencialidades y limitaciones que contiene la inclusión social mediante la presentación del contexto de su surgimiento, sus principales características y sus implicancias analíticas. Se destaca aquí que la inclusión no solo refiere a un término diferente en la nomenclatura de las políticas públicas, sino que también influye sobre la forma que tienen los programas y planes para abordar las problemáticas sociales, y sobre su vinculación con el paradigma de la desigualdad.

\section{Palabras clave}

Inclusión social, políticas públicas, sociedad, bienestar, desigualdad

Reflections on the potential and limitations of social inclusion

\section{ABSTRACT}

The main purpose of this paper is to contribute to the debate on the notion of social inclusion, which in recent decades has emerged as a central objective of Public Policies, associated with a positive connotation. While social inclusion is characterized by its high degree of political and social acceptance, in academia its

* Licenciado en Economía (UNLP). Becario doctoral del Consejo Nacional de Investigaciones Científicas y Técnicas (CONICET, Argentina), Programa de Estudios Regionales y Territoriales, Instituto de Geografía Dr. Romualdo Ardissone, Facultad de Filosofía y Letras, Universidad de Buenos Aires. Correo electrónico: lisandrofernandez85@gmail.com. 
excessive level of generality and lack of theoretical density is pointed out. Against this background, the article makes a theoretical reflection about the potentialities and limitations contained in 'social inclusion', by presenting the context of its emergence, its main characteristics, and its analytical implications. It stresses that inclusion, not only refers to a different term in the nomenclature of public policies, but it also influences the manner in which the Plans and Programs address social issues, and its linkage with the paradigm of inequality.

\section{Keywords}

Social inclusion, public policy, society, welfare, inequality

\section{Introducción}

Desde finales del siglo XX y principios del siglo XXI, la noción de inclusión social cobró amplia difusión como uno de los objetivos centrales de las políticas públicas en Europa y América Latina, asociada a un ideario con fuerte impronta positiva (Atkinson, 2004; Grassi, 2008). Uno de los motivos del uso extendido de la distinción inclusión/exclusión social en el diseño de políticas reside en que permite introducir un criterio estratégico-político fácilmente entendible y transversalmente aplicable: referido a diversos sectores de la población y múltiples problemáticas sociales (Mascareño y Carvajal, 2015).

A lo largo de las últimas décadas, la inclusión social se convirtió en un imperativo para la construcción de muchas políticas sociales, que serviría por sí misma para explicar su implementación. Este resultado político-discursivo se sostiene en tres argumentos que presentan la inclusión como (i) 'natural', (ii) un 'bien en sí mismo' (iii) y una cuestión 'necesaria' (Estermann Meyer, Dal'Igna, Klein y Da Cunha Silveira, 2014). Debido a ello, implica una noción que puede ser declamada por un amplio espectro del arco ideológico.

Al mismo tiempo que la inclusión representa un término utilizado cotidianamente en el discurso y la publicidad política, se destaca que también instituye procesos y modos de organizar el trabajo en las instituciones involucradas para tal fin (Rambla y Jacovkis, 2011; Estermann Meyer et al., 2014).

Sin embargo, lo que en principio resulta una potencialidad para la fundamentación de las políticas sociales (ya que la inclusión social dispone de elevados grados de aceptación y es fácilmente entendible), contrariamente representa una fuerte limitación en el campo académico, dentro del cual la noción es señalada por su escasa "densidad teórica" (Grassi, 2008, p. 146). En efecto, una de las virtudes 
de la perspectiva de la inclusión social es su carácter multidimensional, que permite complejizar los análisis de las diversas problemáticas sociales que afectan a distintos sectores de la sociedad, pero al mismo tiempo opaca el debate acerca de la importancia relativa de cada esfera o área del bienestar social, y la desigualdad en las jerarquías verticales de la estructura social. Por eso, se considera necesario realizar un análisis de reflexión teórica sobre la noción de inclusión social, presentando sus potencialidades y limitaciones con el fin de contribuir al debate en la temática, sin por ello pretender establecer una evaluación o conclusión definitiva.

El presente trabajo se organiza de la siguiente manera. Luego de esta introducción, en la sección siguiente, se realiza una breve presentación del término inclusión social. En la tercera sección, se analiza el contexto histórico-geográfico en el que se difunde, su vínculo con las políticas públicas y las principales consecuencias analíticas. En la cuarta sección, se problematiza el carácter multidimensional de la perspectiva objeto de estudio, resaltando las ventajas para el estudio de fenómenos sociales que se enlazan, pero también sus problemas en términos sociológicos. En la quinta sección, se reflexiona sobre el desplazamiento de la cuestión social que implica el clivaje horizontal de la inclusión social, y su relación con los tópicos de igualdad/desigualdad. Por último, el trabajo finaliza con las consideraciones finales.

\section{Una noción polisémica}

La inclusión social es utilizada en muchas ocasiones con excesiva laxitud y referida a múltiples políticas, acciones públicas o experiencias sociales, pero resulta dificultoso hallar una definición conceptual comúnmente aceptada que sea de consenso en términos académicos. Incluso, es habitual señalar su carácter ambiguo e impreciso.

Como punto de partida para el acercamiento a las principales características del enfoque de la inclusión, es conveniente en primer lugar comenzar por plantear su diferencia con el concepto de integración social, en ocasiones presentados ambos términos como equivalentes. Al respecto, Grassi (2008) indica que desde el siglo XIX el problema de la integración es un antiguo tema de la ciencia social, referido a la posibilidad de existencia de una 'unidad social autoidentificada'. Más tarde, en el siglo XX, muy vinculado a la conformación de los Estados de Bienestar, el desarrollo de estos debates se relacionó con una visión organicista de los problemas de la integración, que desestimaba el conflicto social. En concreto, las políticas de integración se asociaron con el reconocimiento general de los derechos vinculados con la protección social de todos los ciudadanos (De la Cal Barredo y De la Fuente Lavín, 2010). 
Este proceso de discusión sobre modelos de sociedad y políticas públicas repercutió en el ámbito de las ciencias sociales, en el que las preocupaciones por la integración fueron mutando luego de mediados del siglo XX hasta sustituirse por las de inclusión, que no se condicen necesariamente con la integración, entendida esta como "participación y mutuo reconocimiento" (Grassi, 2008, p. 147). Por el contrario, como señalan De la Cal Barredo y De la Fuente Lavín (2010), la inclusión social tendría una referencia más acotada, ya que alude de manera más restringida a ciertos grupos de personas o sujetos, no a la ciudadanía en general. De la mano de este cambio, la referencia deja de situarse en las relaciones sociales y las problemáticas derivadas de ellas, y pasa a enfocarse en las características de los individuos.

Paulatinamente, la inclusión social pasó a predominar en las consideraciones políticas, especialmente desde la década de 1970, y con mayor protagonismo a partir de finales del siglo XX, aunque sin una precisión teórica específica. Algunas de las definiciones dan cuenta del alto grado de generalización e indefinición que las caracterizan en su aspecto teórico. A modo de ejemplo, se mencionarán cuatro definiciones explícitas. Por un lado, Lo Vuolo (1995) afirmó que "[l]a inclusión social significa englobar al conjunto de la población en el sistema de instituciones sociales, concierne tanto al acceso a sus beneficios, como a la dependencia del modo de vida individual con respecto a los mismos" (1995, p. 15).

Por otro lado, se la suele asociar con la distribución de la riqueza y el bienestar, aunque con elevados niveles de imprecisión. Para Ocampo (2004), la inclusión social se asocia con el "énfasis en cómo distribuir más equitativamente los beneficios del desarrollo, las redes de interacción social y la participación política” (2004, p. 37). De manera similar, en un documento oficial del Ministerio de Desarrollo Social de Argentina (2010), se afirma que "[la inclusión social] consiste en la implementación de políticas públicas encaminadas a la vinculación de todos los miembros de la sociedad para la participación de los beneficios que ésta adquiere" (2010, p. 53). Y, más recientemente, Oxoby (2009) afirma:

Inclusión es un aspecto acerca de cómo perciben el acceso a las instituciones y recursos en un entorno de toma de decisiones. Como tal, la inclusión afecta la manera en que los individuos perciben los retornos en varias formas de capital. (2009, p. 7; traducción mía)

No obstante, la indeterminación que exhiben las definiciones precedentes representa una debilidad en el campo académico, pero ello no impide que la inclusión social sea asociada a una connotación o sentido positivo, revestido de "buenos 
sentimientos" (Grassi, 2008, p. 147), por lo que puede ser declamada por un amplio arco ideológico, especialmente en el ámbito de las políticas públicas (Atkinson, 2004). En efecto, en el campo de las políticas sociales la inclusión social ha sido asumida como un imperativo necesario que fundamenta por sí mismo la implementación de aquellas (Estermann Meyer et al., 2014). Por lo pronto, existe un contraste entre la insuficiencia o las limitaciones de los sistemas conceptuales y analíticos en torno a la temática, y el crecimiento de las políticas sociales que invocan la inclusión. Sobre este punto, Raya Diez (2007) plantea la hipótesis de que existe cierta intencionalidad o conveniencia política en mantener la ambigüedad en las definiciones "para dar la sensación de que cualquier política que se encamine hacia la lucha contra la exclusión social responde a un objetivo 'políticamente correcto', por escaso que sea, puesto que difícilmente pueda solventar el problema en su conjunto" (2007, p. 159). Esta hipótesis deja interrogantes abiertos acerca de qué tipo de intereses pueden motivar dicha intencionalidad política y a qué actores sociales responden.

El presente trabajo no pretende dar respuesta categórica a dicho planteo (que merece un análisis específico de situaciones concretas), pero sí realizar una contribución acerca del vínculo entre la noción de inclusión social y las políticas públicas desde una reflexión teórica. A continuación, se indagará sobre los orígenes de esta relación, con el fin de plantear los factores que influyeron en ella. Si bien el interés principal se sitúa en el concepto de inclusión social, no puede dejar de hacerse referencia a su contraparte, la exclusión, por ser la representación de lo que se busca solucionar invocando a aquella.

Surgimiento del concepto de inclusión: contexto geográfico y social. El 'contrato' entre el individuo y la sociedad

El origen de la discusión acerca de diversos fenómenos sociales en términos de inclusión y exclusión social se sitúa en la sociología francesa de la década de 1970, ante la emergencia de problemas sociales experimentados en Europa, asumidos desde la perspectiva de la fragilidad y vulnerabilidad sociales. Una de las primeras referencias es el trabajo titulado Los excluidos. Un francés sobre diez, de René Lenoir (1974), quien acuñó el término 'excluidos' en relación con las personas con discapacidades, enfermedades mentales, toxicómanos, huérfanos, incluso ancianos y familias monoparentales; en síntesis, todos aquellos que en un momento de expansión económica quedaban excluidos del progreso (Castel, 1997; Kessler, 2011; Mascareño y Carvajal, 2015). 
Posteriormente, con la caída del Estado de Bienestar y la pérdida del pleno empleo en los países europeos a partir de los años 80 , nuevos fenómenos sociales (precarización y flexibilidad laboral, desocupación, discriminación de género, etc.) comenzaron a expandirse y tener mayor relevancia en las preocupaciones políticas y académicas. Se hizo necesario, entonces, tomar en cuenta los diversos factores relacionados entre sí que afectaban a las personas: empleo, educación, salud, vivienda, relaciones sociales, entre otros. De este modo, fueron creciendo los cuestionamientos al concepto unidimensional de pobreza entendida exclusivamente en términos de umbrales de ingresos como preocupación central, debido a la diversidad de problemáticas sociales que la excedían (Lope Peña, 2015). Ante ello, se postuló la noción de exclusión social como un concepto multidimensional superador, que concibe las nuevas problemáticas en términos de privaciones para ejercer de forma efectiva los derechos políticos y sociales propios del conjunto de la ciudadanía (Subirats et al., 2004).

La exclusión social pasa a definirse como una situación concreta, fruto de un proceso dinámico de acumulación, superposición y/o combinación de diversos factores de desventaja o vulnerabilidad social que pueden afectar a personas o grupos, generando una situación de imposibilidad de acceder a los mecanismos de desarrollo personal y a los sistemas preestablecidos de protección social (Subirats et al., 2004). En ese sentido, Kessler (2011) puntualiza que la diferencia entre pobreza y exclusión está en el hincapié puesto en la participación social, el reconocimiento social o en las relaciones sociales que pondera la segunda.

Fue así que al quebrarse la articulación entre trabajo y protección desde mediados de la década de 1970, se puso en crisis la relación que garantizaba la inclusión social y el ejercicio pleno de la ciudadanía por medio del trabajo (Castel, 1997; Lope Peña, 2015). Por este motivo, se coincide con Grassi (2008) cuando afirma que "la noción de inclusión parece más acorde con un contexto o red conceptual con la que es afín la idea [...] del fin del trabajo" (2008, p. 146; cursivas en el original).

En definitiva, una vez instalado el tema de la exclusión en la agenda pública bajo una clara connotación negativa, su reverso, la inclusión, se insertó en las metas y objetivos de la formulación e implementación de políticas públicas representando, por contraposición, un ideario con fuerte impronta positiva (Atkinson, 2004).

Años después, durante el período marcado por el fin del siglo XX y principios del XXI, la Unión Europea (UE) explicitó como objetivo de política pública la inclusión social, aunque ya venía implementando en forma conjunta distintos programas de lucha contra la pobreza en los países miembros. Concretamente, en las cumbres de Lisboa y Niza del año 2000, la UE impulsó una serie de 'Planes Nacionales de Inclusión Social', en los cuales instaban a los países miembros a 
diseñar y aprobar planes nacionales para enfrentar los retos de la 'cohesión social' (Subirats et al., 2004). Estos planes tenían una duración prevista de dos años (2001-2003), que luego fueron reformulados y ampliados en una segunda ronda (años 2003-2005). Los objetivos de los Planes Nacionales estaban vinculados al acceso al empleo y diversos recursos, derechos, bienes y servicios de todos los ciudadanos; la prevención de los riesgos de exclusión y la actuación en favor de los más vulnerables. Más allá de las dificultades para establecer comparaciones metodológicas, se definieron diez indicadores primarios comunes para la evaluación de los planes de inclusión social (Atkinson, 2004; Oxoby, 2009).

No obstante, esta propuesta pareciera haber avanzado más en el plano de la formulación que en la implementación. Según Subirats et al. (2004), el nivel de concreción de los planes ha sido muy disparejo entre los países miembros de la UE, ya que según la propia categorización de estos autores, solo tres países: Alemania, España y Finlandia, tuvieron 'alto nivel' de concreción de las medidas propuestas por los planes de inclusión social.

Más tarde, en 2008, en un contexto de crisis económica internacional, la UE reconoció mediante una recomendación de la Comisión Europea la "Estrategia de Inclusión Activa" en favor de las personas consideradas excluidas del mercado de trabajo. Dicho planteo se basaba en tres ejes: (i) una adecuada política de rentas mínimas, (ii) un mercado de trabajo inclusivo y (iii) el acceso a servicios de calidad (De la Cal Barredo y De la Fuente Lavín, 2010). Sin embargo, la estrategia de inclusión activa prioriza la inserción laboral como mecanismo de lucha contra la pobreza y la exclusión social, ya que enfatiza la función integradora del trabajo remunerado y el rol en torno a la participación y la inserción social.

En paralelo, con los primeros años del siglo XXI, organismos internacionales como el Banco Interamericano de Desarrollo (BID), el Banco Mundial (BM), las Naciones Unidas y la CEPAL, tomaron la idea de inclusión social incorporándola a su agenda y, desde entonces, la difundieron en América Latina (Buvinić, 2004), utilizando como vías sus documentos de investigación, reuniones específicas de concertación y la introducción en la gestión de programas sociales que estos mismos financian (Jarque, 2004; Zalakain, 2013).

Desde la perspectiva impulsada por estos organismos, la inclusión social es entendida en términos de 'manejo de riesgos sociales' que afectan al 'grupo beneficiario' de sus programas (Rambla y Jacovkis, 2011). Esos riesgos hacen alusión a la falta de ingresos o a los problemas de salud, pero asimilados a situaciones de 'emergencia' (sin referencia a las relaciones sociales ni a los actores que la componen), con lo cual la exclusión social termina funcionando como una adversidad sin nombre (Vakaloulis, 2000), o, en términos de Zalakain (2013), esta concepción 
implica la desocialización de las causas de la pobreza, ya que los procesos y actores sociales involucrados quedan marginados del debate y análisis.

Ante los problemas de pobreza, desocupación y otros, las recomendaciones de los organismos internacionales pasan por acciones de inclusión desde una gestión multidimensional, pero poniendo el foco en el propio comportamiento de los receptores del programa para superar tales riesgos sociales. Por ende, su centro de atención e intervención se focaliza en el cambio de las conductas, las motivaciones y los comportamientos individuales, antes que en favorecer las condiciones políticas adecuadas para una justa redistribución de la riqueza (Rambla y Jacovkis, 2011). De este modo, las políticas sociales se orientan a 'preparar' a la población para prevenir ciertos 'riesgos' relacionados con la mayor inestabilidad laboral y familiar de las sociedades contemporáneas (Zalakain, 2013).

Lo que interesa resaltar aquí es que, de forma continua y progresiva, el discurso de la inclusión social fue ganando terreno hasta el punto de instituir modos de organizar el trabajo dentro de los organismos (ministerios y trabajadores) implicados en las tareas cotidianas de las políticas sociales (Estermann Meyer et al., 2014). A modo de ejemplo, según Rambla y Jacovkis (2011), el discurso de los organismos internacionales impregnó la formulación e implementación del Programa Familias por la Inclusión Social de Argentina, especialmente en relación con la concepción de la inclusión en términos de (i) los riesgos sociales, (ii) el grupo beneficiario, (iii) las acciones de inclusión, y (iv) la evaluación y gestión del programa.

Otro aspecto relevante es la individualización que conlleva el surgimiento de la idea de inclusión social. En efecto, según el Consejo de Europa (2001), su 'innovación' reside en su asociación con la noción de inserción, que denota un 'contrato' de las políticas sociales entre las responsabilidades individuales del beneficiario y las de la sociedad en su conjunto. Este paradigma es denominado como "principio de la contractualización" por Zalakain (2013, p. 186), y está regulado por una 'norma de reciprocidad' entre el individuo y la sociedad. En palabras de Castel (1997), esta propuesta se englobó en un contexto en los países europeos marcado por "el pasaje de politicas llevadas a cabo en nombre de la integración, hasta politicas conducidas en nombre de la inserción" (1997, p. 422; cursivas en el original). Entre las primeras, el autor sitúa las acciones animadas por la búsqueda de equilibrios y la homogenización de la sociedad. En cambio, las segundas obedecen a una lógica de 'discriminación positiva', ya que se focalizan en poblaciones particulares y zonas singulares del espacio social, desplegando estrategias específicas para ellas.

De este modo, la referencia a la solidaridad como legitimadora de la acción pública es reemplazada por el énfasis en la responsabilidad del individuo. En este esquema, el 'contrato' implica un cambio en los términos en los que se piensa la 
ciudadanía, ya que el acceso de los ciudadanos a sus derechos pasa a ser condicional, dependiendo de su actitud y comportamiento, convirtiéndola en una cuestión de 'merecimiento' (Zalakain, 2013).

Se advierte, entonces, que la idea de exclusión social surgida en Europa intentó dar respuesta a los cambios sociales y económicos ocurridos desde mediados de los años ochenta en dicho continente. De la mano de esta propuesta, la idea de inclusión social ligada a las políticas públicas formuladas para dar respuesta a la 'nueva cuestión social', no solo resultó ser un nuevo término en la nomenclatura política, sino que también implicó un determinado abordaje acerca de las crecientes problemáticas sociales de fines del siglo XX, y sobre la forma de entender la relación individuo/sociedad. Esto requiere profundizar la reflexión sobre sus rasgos distintivos.

\section{Una perspectiva multidimensional}

Del mismo modo en que el concepto de exclusión social se asumía superador de la idea de pobreza por tener en consideración la diversidad de privaciones que pueden afectar a una persona y no exclusivamente la falta de ingresos, también se resalta el carácter multidimensional de la inclusión social, vinculado a las diferentes áreas o esferas que componen el bienestar social. Asimismo, la variedad de componentes para el análisis agrega un mayor nivel de complejidad para la identificación de situaciones de inclusión. Como señalan Mascareño y Carvajal (2015), en muchas ocasiones el par inclusión/exclusión fue tratado en términos binarios: las personas están 'adentro o afuera' de ciertos criterios específicos de la consideración social. Por ejemplo, Lo Vuolo (1995) entiende que "[l]a exclusión en la sociedad [...] es un fenómeno claramente dicotómico y sólo puede entenderse adecuadamente en relación con su opuesto: la noción de inclusión en la sociedad...” (1995, p. 15). En coincidencia, Cadenas (2012) señala que, en términos abstractos, la inclusión/ exclusión corresponde a una "forma de dos lados" (2012, p. 63) que divide el mundo mediante una esquematización en dos opciones claramente separadas: la inclusión o la exclusión.

Si bien esta fórmula binaria puede ser útil en el diseño de políticas, en tanto aporta orientaciones concretas para las intervenciones y para mostrar resultados, desde un punto de vista sociológico una diferencia categórica entre inclusión y exclusión parece más una ilusión con finalidades instrumentales que una observación de acontecimientos sociales reales, ya que oculta paradojas (Mascareño y Carvajal, 2015). 
Se debe tener en cuenta que la inclusión social se inscribe en un contexto en ciencias sociales en donde se resalta la contingencia y el carácter relacional del sujeto colectivo, que favorece la diversidad de espacios para el accionar político (Amaral y Burity, 2006). Respecto de esto, Mascareño y Carvajal (2015) analizan el recorrido del paradigma inclusión/exclusión a través de diferentes corrientes sociológicas como la funcionalista (Durkheim, Simmel, Parsons), la tradición sistémica (Luhmann, Stichweh) y la escuela francesa (Fitoussi y Rosanvallon), lo cual los lleva a dar cuenta de la complejidad de situaciones que contiene, las paradojas reales que visibilizan y la diversidad de posibilidades descriptivas que permite. Así, los autores concluyen que las personas no están incluidas o excluidas de manera absoluta, sino que, en la situación concreta en la que operan, están en ambos 'lugares' a la vez. Por eso la inclusión involucra la idea de pluralidad de campos de acción, de espacios de negociación de conflictos e interlocutores (Calderón, 2007).

La perspectiva señalada encuentra sustento en el esquema de capacidades de Sen (2000), para quien la riqueza medida en ingresos no es deseable por sí misma, sino por el conjunto de capacidades que otorga a las personas para llevar el tipo de vida que valoran y tienen razones para apreciar, buscando ampliar las oportunidades que disponen los individuos (Oxoby, 2009). De este modo, se está en presencia de un enfoque pluralista, dado que existe una gran complejidad en otorgar un peso mayor a ciertas capacidades por sobre otras (Sen, 2000), lo cual puede generar controversias a la hora de determinar qué esferas del bienestar se deben ponderar (Kessler, 2011). Desde este marco teórico, la inclusión social sirve entonces para promover capacidades humanas en sus múltiples dimensiones (Calderón, 2007) y las intervenciones estatales pueden ser interpretadas como eliminación de las privaciones de capacidades en diversas dimensiones del orden social. No obstante, queda abierto el interrogante acerca de cuál es la cuantía precisa de acumulación de privaciones en las múltiples dimensiones de la inclusión, que da por resultado la exclusión social de una persona o grupo social y, al mismo tiempo, si es posible (o no) determinarla.

Para Zalakain (2013), "la multidimensionalidad de la inclusión, [implica] asumir que, en función de las necesidades y situaciones individuales, la inclusión puede alcanzarse desde muy diversos ámbitos vitales, y no solo desde la inclusión laboral" (2013, p. 200). Esta afirmación resalta la influencia del origen del paradigma en el cual, como se señaló más arriba, se quebró la relación unívoca entre trabajo e inclusión social. Esto no implica que el empleo no sea importante, sino que ya no es suficiente para evaluar la inclusión de una persona o grupo social: se requiere tener en cuenta otros factores. 
Aunque no existe un consenso generalizado sobre qué y cuántas variables conforman la base de la inclusión, a continuación se presenta el esquema propuesto por Subirats et al. (2004), en el cual la multidimensionalidad de la inclusión social pasa por la inserción de las personas en tres ejes básicos, que en ningún caso pueden ser analizados de manera hermética:

\section{Esquema 1: Pilares de la inclusión social}

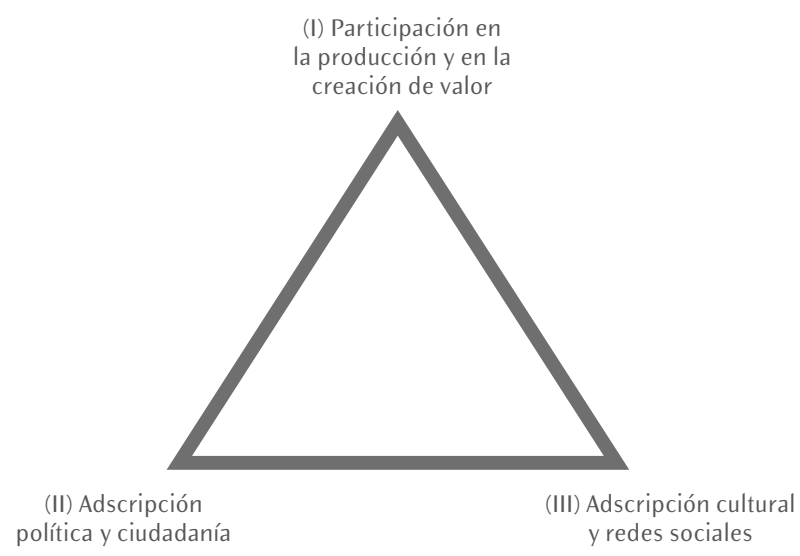

Fuente: Subirats et al. (2004).

La inclusión de las personas o grupos en dichas áreas se traduce (Subirats et al., 2004; De la Cal Barredo y De la Fuente Lavín, 2010) en:

- (I) El mercado y la utilidad social aportada por cada persona como mecanismo de intercambio y de vinculación con la creación de valor. Aquí se refiere fundamentalmente a la participación en el mercado de trabajo. - (II) La redistribución que llevan a cabo las administraciones públicas mediante la concesión de transferencias y la provisión de servicios públicos. - (III) Las relaciones de reciprocidad que se despliegan en el marco de la familia y las redes sociales, y que proporcionan apoyo y ayuda.

El esquema se articula con la propuesta realizada por Subirats junto a otros autores en Laparra et al. (2007), texto en el que resaltan las dimensiones (a) económicas, (b) políticas y (c) sociales que conforman la exclusión social, las que (se puede inferir) se corresponden con los pilares (I), (II) y (III) del triángulo. 
Este diseño conceptual permite graficar las diversas esferas de la inclusión social por separado, pero de forma interrelacionada. Por ejemplo, el acceso a un tipo de empleo particular (por caso formal) se vincula con determinado acceso a derechos laborales otorgados por el Estado y a un tipo de redes sociales, lo que constituye el 'capital relacional' o 'capital social'. Por el contrario, las desigualdades generadas por la escala jerárquica vertical en el mercado laboral, se ven reforzadas por la descomposición del Estado de Bienestar, y eso repercute en los vínculos sociales (Subirats et al., 2004).

Se coincide con Kessler (2011) acerca de que la discusión en torno a cuáles son las dimensiones o pilares prioritarios de la inclusión (y exclusión) social redunda en un debate político. No obstante, a modo de ejemplo, en el caso del Viejo Continente, el Consejo de Europa distinguió cinco grandes secciones para el fomento de la inclusión en las políticas públicas planteadas en términos de acceso al: empleo, la vivienda, la protección social, la salud y la educación. En cada una de ellas, el eje transversal de las intervenciones estatales está en la promoción del acceso a las oportunidades y elecciones individuales en el largo plazo (Oxoby, 2009).

En definitiva, importa resaltar que la multiplicidad de dimensiones posibles de análisis genera que la distinción binaria inclusión/exclusión quede obsoleta, porque desde este enfoque difícilmente existe un estado único o completo de inclusión o exclusión, sino situaciones concretas en donde ambas condiciones se manifiestan al mismo tiempo. Para sortear este dilema, Mascareño y Carvajal (2015) proponen cinco 'constelaciones' en las que las situaciones de inclusión y exclusión se entrelazan según el grado de selectividad individual y el contexto organizacional:

(i) Autoinclusión: situación en la que los individuos eligen participar o no participar en algún rendimiento social.

(ii) Inclusión por riesgo: en la que los individuos pueden estar en peligro por las decisiones que tomen las organizaciones a las que pertenecen.

(iii) Inclusión compensatoria: aquella que instituciones sociales llevan adelante (mediante políticas públicas, subsidios, legislación o acciones ad hoc) para equilibrar situaciones que se asumen temporales;

(iv) Inclusión en la exclusión: implica una condición de inclusión, pero en una posición de subordinación en comparación con otras categorías sociales.

(v) Subinclusión: supone la ausencia de condiciones institucionales para hacer valer derechos fundamentales, pero la obligación de responder a un orden social que exige obediencia. 
Esta clasificación es una de las posibilidades mediante la cual se puede conceptualizar el acoplamiento entre situaciones de inclusión y exclusión social, producto de la multidimensionalidad que ambas exhiben. Más allá de la misma, interesa señalar que la existencia de múltiples esferas por las que se canaliza la inclusión, genera condiciones de posibilidad para que una misma persona o grupo social pueda estar incluido desde la perspectiva de una dimensión de análisis, pero, excluido desde otra. Como consecuencia de ello, se soslaya la importancia de los principios de estratificación social; por ejemplo, los que se basan en las clases sociales. Esto es, si se asume el análisis de las problemáticas sociales desde el paradigma de la inclusión, personas de una misma clase social pueden percibir cada una privaciones en diversas dimensiones $y$, al mismo tiempo, personas de diferentes clases pueden percibir privaciones de la misma esfera de análisis, por lo cual esta docilidad analítica relativiza las desigualdades de jerarquía vertical en la estructura social (Vakaloulis, 2000).

Sobre este punto, Mascareño y Carvajal (2015) difieren cuando señalan que el proceso de inclusión no implica la desaparición de las modalidades de estratificación y jerarquización clásicas, sino una 'fuerte individualización de sus formas', tornando inestables las referencias al estatus de un grupo, una clase o una comunidad. En un sentido similar, basado en el enfoque de la teoría de los sistemas sociales, Cadenas (2012) sostiene que la forma inclusión/exclusión no sustituye a la forma igualdad/desigualdad como elemento constitutivo de la diferenciación social, y afirma que tanto en la inclusión como en la exclusión hay desigualdades. Aquí se encuentra un debate que requiere ser profundizado.

\section{El desplazamiento de la cuestión social}

Si bien la perspectiva multidimensional que compone la inclusión social permite ampliar y complejizar los factores y problemáticas que afectan a una persona más allá de su nivel de ingresos o su situación laboral, se debe señalar que la misma implica un cambio analítico: esto es, además de la individualización de las problemáticas sociales, también conlleva el desplazamiento de la cuestión social centrada en el 'updown', propio de las desigualdades de estratificación vertical, donde los que están en la base inferior intentan revertir o acortar la pirámide a otra centrada en el 'in-out' (Kessler, 2011), donde los que están 'adentro' procuran conservar su lugar, mientras que los que están 'afuera' pugnan por su inclusión, y así alcanzar el lugar central.

A partir de lo antes descrito se adopta un clivaje horizontal (Vakaloulis, 2000), en el que los factores que favorecen o perjudican la inclusión social son vistos como la sumatoria de diversas capacidades que sitúan a las personas más lejos o más cerca 
del centro que aglutina al orden social (Laparra et al., 2007). Así, el hecho de soslayar las jerarquías verticales implica relegar o soslayar los debates en torno a la desigualdad, que tiene en su centro mismo una diferencia, y de la mano de ello, también la categoría clase social como un factor explicativo de las posibilidades de acceso a bienes y servicios, $\mathrm{y}$ trayectorias de vida.

Aunque este clivaje horizontal incluye y permite contemplar múltiples áreas o dimensiones que pueden enriquecer un análisis concreto, introduce cierto grado de imprecisión conceptual, ya que como se analizó en el apartado previo, las 'fronteras' entre inclusión/exclusión son cada vez más móviles: la inclusión social analiza las causas de los procesos sociales como una heterogeneidad de espacios situados en "el continuo entre integración y exclusión" (Laparra et al., 2007, p. 29).

En el mismo sentido, Kessler (2011) coincide cuando refiere que "en el caso de muchos países latinoamericanos es innegable la persistencia de sectores totalmente excluidos pero el panorama actual muestra una complejidad mayor, en el que distintos sectores están excluidos de algunas esferas pero permanecen incluidos en otras" (2011, p. 9). No obstante, advierte que es preciso jerarquizar las esferas, ya que no es indiferente estar excluido o incluido en el sistema de salud o de vivienda que en la esfera del esparcimiento. De todos modos, dicha decisión se vincula con los intereses con que se realizan los estudios específicos o con la toma de posición de quienes formulan e implementan las políticas públicas que invocan la inclusión social como objetivo central.

$\mathrm{Al}$ mismo tiempo, queda abierto el interrogante acerca de los gradientes posibles dentro de cada una de las dimensiones que componen la inclusión, y en qué momento la desigualdad entre personas o grupos en una misma esfera puede devenir en exclusión. Esta cuestión queda velada en tanto que, el enfoque de capacidades antes descrito, posee un fuerte planteo individualista, que sitúa el foco en las habilidades o potencialidades de las personas, cuando el análisis de la desigualdad implica una mirada relacional (ya sea entre personas o grupos sociales). Por ende, como una de las derivaciones de la perspectiva de las capacidades, se emplaza el interés en cómo transformar las características individuales para que las personas lleguen a la 'zona de inclusión' antes que en las relaciones sociales que generan desigualdad (Laparra et al., 2007; Grassi, 2008; Zalakain, 2013), por lo cual la exclusión social termina funcionando como una 'adversidad sin nombre' (Vakaloulis, 2000). Conjuntamente, quedan en segundo plano las desigualdades verticales, o cuanto menos se ocultan o matizan tanto el aspecto relacional como los actores sociales y los factores que están en el origen de la desigualdad.

La influencia de esta perspectiva en las políticas públicas puede advertirse (por ejemplo) en los programas que postulan entre sus objetivos fomentar la capacitación 
o la 'empleabilidad' de los individuos. En efecto, con el argumento de sustituir las políticas 'pasivas' en pos de políticas guiadas por el 'paradigma de la activación', en las que las intervenciones se personalizan cada vez más, tratando de influir en los comportamientos del individuo frente al mercado de trabajo y actuando sobre las conductas, motivaciones y actitudes individuales (De la Cal Barredo y De la Fuente Lavín, 2010; Zalakain, 2013).

Sin embargo, desde una mirada crítica sobre el énfasis en la empleabilidad, Grassi (2008) advierte que los saberes, habilidades y disposiciones para trabajar no se encuentran en la naturaleza, ni se cargan como un soft a los destinatarios de los programas de capacitación o empleo, sino que las mismas se producen y reproducen socialmente. Por eso, la disponibilidad de ciertas capacidades laborales no depende de las voluntades individuales, sino en mayor medida de las decisiones y acciones de estrategias empresariales y públicas, que darán continuidad (o no) en distintos sectores de la economía. De todas formas, se debe señalar que las políticas de inclusión social guiadas por el paradigma de la activación, se corresponden con el 'principio de la contractualización' de Zalakain (2013) apuntado más arriba, por el cual se otorga un peso específico a las responsabilidades individuales en la búsqueda de empleo por sobre la dinámica propia de la economía y la influencia de las políticas públicas en el acceso al trabajo.

La individualización que supone la inclusión social desplaza entonces del centro del debate las relaciones sociales, y con ellas los antagonismos y las luchas, que quedan dispersos y, por ende, las demandas de inclusión se cruzan cada vez más con la cuestión de la afirmación de la diferencia, las políticas de reconocimiento y la promoción de la diversidad (Calderón, 2007). Esto genera que, en muchas ocasiones, la connotación positiva en el discurso de la inclusión social puede asociarse con la posibilidad de conseguir mejoras económicas sin conflictos, en donde todos los sectores sociales mejoran su situación socioeconómica al mismo tiempo.

Este esquema se vincula con el paradigma de la solidaridad 'durkheimiana', que a lo largo del siglo XX, cuando los problemas de la integración social se trasladaron hacia la inclusión social, adoptaron una concepción organicista, que entiende el Estado como un agente activo capaz de generar las condiciones para adaptar a la población a los imperativos de la modernización (Grassi, 2008). De este modo, en la búsqueda de 'cohesión social' en las políticas públicas subyace la idea de gestión estatal del desorden a combatir (Vakaloulis, 2000): si la exclusión es un debilitamiento de los lazos que mantienen unida a la sociedad, la inclusión designa los esfuerzos intencionales (especialmente del Estado) por sostener esa unidad (Mascareño y Carvajal, 2015). 
Adicionalmente, la referencia positiva y ampliamente aceptada de la inclusión social genera un estándar normativo en la distinción igualdad/desigualdad (ligado a los 'buenos sentimientos', en palabras de Grassi, 2008), asociando la inclusión con la igualdad como principio universal (Mascareño y Carvajal, 2015). Pero, trazando una analogía con Kessler (2014), aquí corresponde la pregunta acerca de igualdad de qué.

Al respecto, Oxoby (2009) afirma que el objetivo último del enfoque de la inclusión social se expresa en la idea de igualdad de oportunidades en la sociedad, propósito que apunta a que todos los individuos tengan igual acceso a las instituciones para desarrollar sus capacidades plenamente y lograr sus objetivos deseados (Salas, 2004). Dicha visión se sustenta en la disputa entre individuos sin ningún desequilibrio en el origen por los lugares más deseables de la estructura social, sistema que toma la competencia como mecanismo de asignación de recursos (Salas, 2004; Kessler, 2014). Se observa entonces que la propuesta de igualar las oportunidades para competir contiene un fuerte sesgo meritocrático, al establecer la distribución de las posiciones sociales en función del mérito de los individuos: ya sea por las habilidades que poseen o por su capacidad de 'usufructuar' las oportunidades brindadas. Quedan relegadas las referencias sobre qué sujetos sociales están en condiciones de aprovechar mejor las oportunidades brindadas y por qué causas.

De este modo, la igualdad de oportunidades que apunta a igualar las posibilidades de acceso a las instituciones que promueven la movilidad social, reconoce a la vez como legítimas las desigualdades de resultados, en tanto estas dependen de las capacidades individuales. Este modelo responde sin dudas a la tradición liberal, por la que la distribución desigual de capacidades, talentos o habilidades que poseen los individuos da sustento a las desigualdades sociales (Salas, 2004). Esto reafirma que, en esta sociedad meritocrática, si los excluidos son aquellas personas que están privados de capacidades, mejorar su capital humano y social a través de reformas se vuelve necesario para aumentar sus posibilidades de inclusión social (Laparra et al., 2007). Sin embargo, debe señalarse que esta estrategia puede coexistir con la permanencia y reproducción de desigualdades (económicas, sociales o políticas) en una sociedad cada vez más compleja.

\section{Consideraciones finales}

A lo largo del presente trabajo se buscó contribuir al debate sobre la inclusión social: una noción de uso extendido en las políticas públicas, de clara connotación positiva, considerada 'bien en sí mismo'. Sin embargo, se señaló que 
su generalizada aceptación en el ámbito de las políticas públicas convive con sus limitaciones en el campo académico, dentro del cual recibe críticas por su escasa 'densidad teórica'. De todos modos, el término inclusión social no solo representa una variación en la nomenclatura de las políticas, sino que también implica nuevas concepciones de las problemáticas sociales y nuevas propuestas de resolución.

Por este motivo se buscó un acercamiento al surgimiento, características e implicancias de la inclusión social y su vínculo con las políticas públicas. Primeramente, se destacó el origen histórico-geográfico del uso de la noción. En un marco de crisis del Estado de Bienestar en Europa desde las décadas de 1970/1980, la expansión de las problemáticas sociales que excedían la cuestión del empleo, repercutieron en los análisis académicos, propiciando una perspectiva multidimensional de la pobreza y del bienestar social. Si bien este cambio permitió complejizar los estudios al aumentar las esferas de observación susceptibles de análisis, al mismo tiempo tuvo repercusiones en la relación individuo/sociedad; prontamente, el término se utilizó para referirse menos a las relaciones sociales y la concepción de una política que a nombrar a las personas y/o grupos sociales calificados como 'excluidos'. De este modo, las intervenciones en pos de la inclusión social se centran en cambiar a los individuos de 'lugar', desplazándolos hacia la 'zona de incluidos', como si se tratara de un movimiento hacia un espacio o totalidad social ya constituida (Laparra et al., 2007; Grassi, 2008).

Por otro lado, también se destacó que el carácter multidimensional de la inclusión social deriva en un clivaje teórico horizontal (Vakaloulis, 2000), en el que no existe un consenso comúnmente aceptado sobre qué dimensiones o esferas del bienestar social deben ser jerarquizadas para los análisis o la elaboración de políticas públicas. Sobre este punto, se coincide con Kessler (2011) cuando advierte que esta discusión es parte de un debate político.

Por último, se destaca que la individualización que conlleva el enfoque de las capacidades, junto a la ilimitada diversificación posible de factores o esferas de análisis, puede marginar de las preocupaciones sociales la importancia de las desigualdades de jerarquía vertical y los principios de estratificación social. Si el ideal de igualdad que promueve la inclusión social se relaciona con la igualdad de oportunidades, como señala Oxoby (2009), la promoción de mayores grados de inclusión social puede ser compatible con la persistencia y reproducción de diferentes tipos de desigualdades en una sociedad.

Al reflexionar de forma crítica en el presente artículo sobre la inclusión social, no se pretendió menoscabar los beneficios económicos o derechos sociales que otorgan las políticas o programas que invocan la inclusión como su objetivo 
central, pero sí poner en consideración más elementos para su valoración. Las reflexiones desarrolladas aquí deberán aplicarse en análisis concretos a fin de ser profundizadas.

Recibido marzo 11, 2016 Aceptado mayo 1, 2016

\section{Referencias bibliográfıcas}

Amaral, A., Burity, J. (2006). Apresentação. En A. Amaral, J. Burity (eds.), Inclusão social. Identidade e diferença. Perspectivas pós-estruturalistas de análise social (pp. 9-18). São Paulo: Annablume.

Atkinson, T. (2004). La experiencia de la Unión Europea con la política de inclusión social. En M. Buvinić, J. Mazza, J. Pungiluppi, R. Deutsch (eds.), Inclusión socialy desarrollo económico en América Latina (pp. 111-142). Washington D.C.: Banco Interamericano de Desarrollo.

Buvinić, M, (2004). Introducción: La inclusión social en América Latina. En M. Buvinić, J. Mazza, J. Pungiluppi, R. Deutsch (eds.), Inclusión social y desarrollo económico en América Latina (pp. 3-35). Washington D.C.: Banco Interamericano de Desarrollo.

Cadenas, H. (2012). La desigualdad en la sociedad. Diferenciación y desigualdad en la sociedad moderna. Persona y Sociedad XXVI (2), 51-77.

Calderón, F (2007). Ciudadanía y desarrollo humano. En F. Calderón (ed.), Ciudadanía y desarrollo humano (pp. 31-63). Buenos Aires: Siglo XXI Editores.

Castel, R. (1997). La metamorfosis de la cuestión social. Una crónica del salariado. Buenos Aires: Paidós.

Consejo de Europa (2001). Promoting the Policy Debate on Social Cohesion from a Comparative Perspective. Trends in Social Cohesion $\mathrm{N}^{\circ} 1$. Alemania: Council of Europe Publishing. Disponible en: http://www.coe.int/t/dg3/socialpolicies/socialcohesiondev/source/Trends/ Trends-01_en.pdf [10 de febrero, 2014].

De la Cal Barredo, M., De la Fuente Lavín, M. (2010). Algunos interrogantes en torno a los programas de inclusión activa como estrategia de lucha contra la pobreza y la exclusión social. Lan Harremanak I (22), 45-87.

Estermann Meyer, D., Dal'Igna, M. C., Klein, C., Da Cunha Silveira, C. (2014). Políticas públicas: imperativos e promesas de inclusão social. Ensaio: Avaliação e Políticas Públicas em Educação, 22 (85), 1001-1026. Disponible en: http://www.scielo.br/pdf/ensaio/v22n85/ v22n85a07.pdf [24 de noviembre, 2014].

Grassi, E (2008). Los olvidos de la política social: reflexiones acerca de la noción y los objetivos de inclusión social. En P. Pavcovich y D. Truccone (eds.), Estudios sobre la pobreza en Argentina (pp. 137-163). Villa María, Córdoba: Eduvim. 
Jarque, C. (2004). Prefacio. En M. Buvinić, J. Mazza, J. Pungiluppi, R. Deutsch (eds.), Inclusión social y desarrollo económico en América Latina (ix-xi). Washington D.C.: Banco Interamericano de Desarrollo.

Kessler, G. (2011). Exclusión social y desigualdad. ¿¿Nociones útiles para pensar la estructura social argentina? Lavboratorio 11 (24), 4-18.

(2014). Controversias sobre la desigualdad. Argentina 2003-2013. Buenos Aires: Fondo de Cultura Económica.

Laparra, M., Obradors, A., Pérez, B., Pérez, M., Renes, V., Sarasa, S., Subirats, J., Trujillo, M. (2007). Una propuesta de consenso sobre el concepto de exclusión. Implicaciones metodológicas. Revista Española del Tercer Sector N 5, enero-abril, 15-57.

Lenoir, R (1974). Les exclus: Un Français sur dix. Paris: Seuil.

Lope Peña, A. (2015). El trabajo y la inclusión social: una relación en peligro. Lavboratorio 15 (26), 107-129.

Lo Vuolo, R. (1995). A modo de presentación: los contenidos de la propuesta de ingreso ciudadano. En Centro interdisciplinario para el Estudio de Políticas Públicas (ed.), Contra la exclusión. La propuesta del ingreso ciudadano (pp. 13-46). Buenos Aires: Miño y Dávila Editores.

Mascareño, A., Carvajal, F. (2015). Los distintos rostros de la inclusión y la exclusión. Revista Cepal 116, 131-146.

Ministerio de Desarrollo Social (2010). Políticas sociales del Bicentenario. Un modelo nacional y popular. Tomo II. Buenos Aires: Ministerio de Desarrollo Social.

Ocampo, J. (2004). Desarrollo económico e inclusión social. En M. Buvinić, J. Mazza, J. Pungiluppi, R. Deutsch (eds.), Inclusión social y desarrollo económico en América Latina (pp. 37-46). Washington D.C.: Banco Interamericano de Desarrollo.

Oxoby, R. (2009). Understanding Social Inclusion, Social Cohesion and Social Capital. Laurier Centre For Economic Research \& Policy Analysis. Economic Research Paper 09. Disponible en: http://econ.ucalgary.ca/sites/econ.ucalgary.ca.manageprofile/files/unitis/ publications/162-34166/UnderstandingSocialInclusion-Oxoby.pdf [31 de octubre, 2013].

Rambla, X., Jacovkis, J. (2011). Entre la gestión y la producción de la pobreza. Un análisis del discurso oficial sobre el Programa Familias para la Inclusión Social en Argentina. Convergencia. Revista en Ciencias Sociales 18 (56), 157-179.

Raya Diez, E. (2007). Exclusión social: Indicadores para su estudio y aplicación para el trabajo social. Revista del Ministerio de Trabajo y Asuntos Sociales (España) 70, 155-172. Disponible en: http://www.empleo.gob.es/es/publica/pub_electronicas/destacadas/revista/ numeros/70/Inf01.pdf [9 de marzo, 2016].

Salas, M. (2004). Desigualdad social: ¿̨nuevos enfoques, viejos dilemas? En M. Mora Salas, J. P. Pérez Sáinz, F. Cortés (eds.), Cuadernos de Ciencias Sociales. Desigualdad social en América Latina 131, 9-44, Flacso, Costa Rica. 
Sen, A. (2000). Desarrollo y libertad. Barcelona: Planeta.

Subirats, J., Riba, C., Giménez, L., Obradors, A., Giménez, M., Queralt, D., Bottos, P., Rapoport, A. (2004). Pobreza y exclusión social. Un análisis de la realidad española y europea. Colección Estudios Sociales 16.

Vakaloulis, M. (2000). Antagonismo social y acción colectiva. Revista del Observatorio Social de América Latina 2 (18), 158-164.

Zalakain, J. (2013). Tendencias y prácticas innovadoras en inclusión social. Perspectiva internacional. Lan Harremanak, II (29), 175-216. 